\section{Optimum Power Control for CDMA With Deterministic Sequences in Fading Channels}

Onur Kaya, Student Member, IEEE, and Sennur Ulukus, Member, IEEE

\begin{abstract}
We specify the capacity region for a power-controlled, fading code-division multiple-access (CDMA) channel. We investigate the properties of the optimum power allocation policy that maximizes the information-theoretic ergodic sum capacity of a CDMA system where the users are assigned arbitrary signature sequences in a frequency flat-fading environment. We provide an iterative waterfilling algorithm to obtain the powers of all users at all channel fade levels, and prove its convergence. Under certain mild conditions on the signature sequences, the optimum power allocation dictates that more than one user transmit simultaneously in some nonzero probability region of the space of all channel states. We identify these conditions, and provide an upper bound on the maximum number of users that can transmit simultaneously at any given time. Using these properties of the sum capacity maximizing power control policy, we also show that the capacity region of the fading CDMA channel is not in general strictly convex.
\end{abstract}

Index Terms-Capacity region, code-division multiple access (CDMA), fading channels, iterative waterfilling, power control, sum capacity.

\section{INTRODUCTION}

Fading may be an important limiting factor in wireless communication networks unless appropriate resource allocation is applied to exploit the variations in the channel gains to the advantage of the network capacity. The resources that we concentrate on allocating optimally in this correspondence are the transmit powers of the users. The quality-of-service-based power control approaches assign transmit powers to the users so that all users satisfy their signal-to-interference-ratio (SIR) requirements while transmitting with the least amount of power. The SIR-based power control assigns powers to the users with the aim of compensating for the variations in the channel; it assigns more power to the users with bad channel states, and less power to the users with good channel states [3]-[6].

For a single-user fading channel, [7] shows that the optimum power allocation policy, in the sense of maximizing the ergodic channel capacity, is a waterfilling of power in time. This policy allocates more power to the stronger channel states, and less power to the weaker channel states; it allocates zero power to the channel states below a threshold level which is determined by the fading statistics.

The capacity of a multiple-access channel (MAC) is expressed as a region of achievable rates [8], [9], and sum capacity, the maximum achievable sum of rates is often used as a measure of the overall network capacity. For a multiuser scalar channel, [10] finds the optimum power allocation policy which maximizes the ergodic sum capacity of the network. For this system, it was shown that the optimum power allocation policy is one where each user compares its channel state (normalized by a factor depending on the statistical characterization of the fading) to those of the other users, and transmits with nonzero power

Manuscript received November 5, 2002; revised December 23, 2003. This work was supported by the National Science Foundation under Grants ANI 02-05330 and CCR 03-11311; and by ARL/CTA under Grant DAAD 19-01-20011. The material in this correspondence was presented in part at the 40th Annual Allerton Conference on Communications, Control and Computing, Monticello, IL, October 2002 [1], and at the IEEE International Symposium on Information Theory, Chicago, IL, June 2004 [2].

The authors are with the Department of Electrical and Computer Engineering, University of Maryland, College Park, MD 20742 USA (e-mail: onurkaya@eng.umd.edu; ulukus@eng.umd.edu).

Communicated by D. N. C. Tse, Asociate Editor for Communications.

Digital Object Identifier 10.1109/TIT.2004.834747 only if its normalized channel state is better than or equal to the normalized channel states of all other users. More than one user transmits simultaneously only if the normalized channel states of multiple users are the same. Since the channel gains are continuous random variables, this occurs only with zero probability. Therefore, this power control policy implies that at most one user transmits (if at all) at any given time with probability one.

While [10] focuses on the sum-rate point on the capacity region of the scalar MAC subject to fading, [11] finds the entire capacity region of such a channel, and specifies the optimal power allocation policies corresponding to each rate tuple on the capacity region.

There has also been some recent work on power control for vector multiple-access channels and their associated capacities. For a multiple-access channel with multiple antennas, [12] solves for the sum capacity maximizing power allocation at all transmit antennas and gives a relationship between the maximum number of active transmit and receive antennas. The problem of maximizing the sum capacity as a function of the transmit powers in a vector multiple-access channel, such as a code-division multiple-access (CDMA) or multiple transmit antenna system, in fading channels, is studied for the case of large systems and random transmit vectors (signature sequences) in [13] where a simple single-user waterfilling strategy is proposed and shown to be asymptotically optimal.

In this correspondence, we focus on a CDMA channel where the number of users and the processing gain are finite and arbitrary, and the users are assigned arbitrary deterministic signature sequences. We first provide the capacity region of a fading CDMA channel where users have perfect channel state information, and are able to choose their transmit powers as a function of these channel states, subject to average power constraints. The capacity region is obtained by a simple extension of [11], which deals with an equivalent problem in the case of scalar MAC.

Like for the scalar fading MAC [11], the capacity region of the fading CDMA is a union of capacity regions obtained for each valid power allocation policy. One of those power allocation policies, namely, the one that maximizes the sum capacity, is worth special attention, both because sum capacity is a commonly used performance metric for multiple-access channels [10], [12], [13], and because it will aid us in investigating the strict convexity of the entire capacity region. Thus, we next focus on the sum capacity maximizing power control policy for a fading CDMA system. Our problem reduces to $K$ independent Goldsmith-Varaiya problems [7] when the signature sequences are chosen to be orthogonal, and to a Knopp-Humblet problem [10] when the signature sequences are chosen to be identical. The optimum power allocation policy is a simultaneous waterfilling policy [12] that requires the solution of a set of highly nonlinear equations. By a simple extension of the iterative algorithm for the nonfading vector MAC of [14] to the fading CDMA channel, we develop an iterative power allocation policy, where, at each step, only one user allocates its power optimally over all channel states of all users when the power allocations of all other users are fixed. The power allocation of each user in this iterative process is a waterfilling where the base level of the water tank is determined by the inverse of the SIR the user would obtain at the output of a minimum mean-squared error (MMSE) receiver if it transmitted with unit power. We prove the convergence of our algorithm to an optimum solution, and provide conditions for the uniqueness of the solution.

One of the questions of interest, for an arbitrary set of signature sequences, is whether there exists a set of channel states having a nonzero probability where either all or some of the users transmit simultaneously. In the case of orthogonal signature sequences, for instance, all users transmit simultaneously in an orthant of the space of all channel 
states where the channel states of all users exceed their corresponding thresholds; and, clearly, this region has a nonzero probability. In the case of identical signature sequences, however, users transmit simultaneously only on a half-line in the space of all channel states; and, this region has a zero probability [10]. In the most general case, the existence of a region of channel states having nonzero probability where all (or more than one) users transmit simultaneously depends on the number of users, the dimensionality of the signal space (processing gain), and the set of signature sequences being used. We identify the conditions under which such a nonzero probability region of channel states exists. These conditions turn out to be very mild; for instance, if the number of users is less than the processing gain and the sequences are linearly independent, a simultaneous transmit region for all users is guaranteed to exist. This region also exists even when the number of users is larger than the processing gain so long as the signature sequences satisfy certain properties. Also, even if these conditions are not satisfied for all users, there may be a subset of users that are guaranteed to transmit simultaneously. This is a result of the fact that the CDMA scheme with nonidentical signature sequences provides users with multiple degrees of freedom; therefore, the users do not have to avoid each other completely in the space of all channel states (as in the case of scalar channels), that is, multiple users can share some of the channel states that are favorable to all of them.

The existence of simultaneous transmit regions is of interest to us for two reasons. First, it serves in proving that the capacity region, unlike its scalar counterpart, is not strictly convex, provided all of the signature sequences are not orthogonal or identical. Second, it provides a sense of fairness, in that while maximizing the overall average rate achieved by the system, it allows users to access the medium more frequently. This is in contrast to the scalar channels where each user has to wait until its channel is the best in order to transmit.

Throughout this correspondence, we will employ the following notation: vectors are represented in bold $(\boldsymbol{x})$, matrices are represented in bold and are capitalized $(\boldsymbol{X})$, and $|\cdot|$ denotes the determinant.

\section{SYSTEM MODEL}

We consider a symbol-synchronous CDMA system with processing gain $N$ where all $K$ users transmit to a single receiver site. In the presence of fading and additive white Gaussian noise (AWGN), the received signal is given by [15]

$$
\boldsymbol{r}=\sum_{i=1}^{K} \sqrt{p_{i} h_{i}} b_{i} \boldsymbol{s}_{i}+\boldsymbol{n}
$$

where, for user $i, b_{i}$ denotes the information symbol with $\mathrm{E}\left[b_{i}^{2}\right]=1$, $\boldsymbol{s}_{i}=\left[s_{i 1}, \ldots, s_{i N}\right]^{\top}$ denotes the unit energy signature sequence, $\sqrt{h_{i}}$ denotes the random and continuously distributed channel gain, and $p_{i}$ denotes the transmit power; $\boldsymbol{n}$ is a zero-mean Gaussian random vector with covariance $\sigma^{2} \boldsymbol{I}_{N}$. We assume that the receiver and all of the transmitters have perfect knowledge of the channel states of all users represented as a vector $\boldsymbol{h}=\left[h_{1}, \ldots, h_{K}\right]^{\top}$, and the components of $\boldsymbol{h}$ are independent. We further assume that although the fading is slow enough to ensure constant channel gain in a symbol interval, it is fast enough so that within the transmission time of a block of symbols the long-term ergodic properties of the fading process can be observed [16].

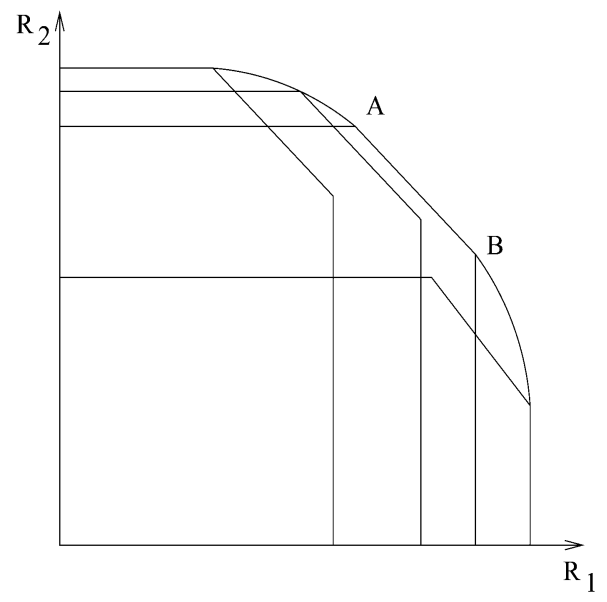

Fig. 1. Sample two-user capacity region.

\section{POWER CONTROL FOR FADING CDMA}

\section{A. Capacity Region With Fixed Sequences and Adaptive Powers}

For the CDMA system given by (1), let the transmitters be able to choose their powers as a function of the channel state, subject to the average power constraints $E_{\boldsymbol{h}}\left[p_{i}(\boldsymbol{h})\right] \leq \bar{p}_{i}$. We first characterize the set of long-term achievable rates, i.e., the capacity region, for fading CDMA. Hanly and Tse [11, Theorem 2.1] have characterized the capacity region for a power-controlled scalar multiple-access channel. Both forward and converse parts of the proof of this theorem can be directly generalized to the CDMA channel, also by incorporating the methods and results from [9, Proposition 1] and [17, Theorem 1]. Therefore, we state the capacity region of the fading CDMA channel in the following theorem, without providing a proof.

Theorem 1: The capacity region of a fading CDMA channel under AWGN, where users have perfect channel state information (CSI) and allocate their powers as a function of the CSI subject to average power constraints $E_{\boldsymbol{h}}\left[p_{i}(\boldsymbol{h})\right] \leq \bar{p}_{i}$ is given by (2) at the bottom of the page.

Fig. 1 illustrates a typical capacity region for some fixed signature sequences $\boldsymbol{s}_{1}$ and $\boldsymbol{s}_{2}$ in a two-user setting. Each of the pentagons correspond to a valid power allocation policy. Note the flat portion on the capacity region, which in fact is the dominant face of one of the pentagons. Unlike scalar multiple-access channel capacity region [11], the capacity region for fading CDMA may contain such a flat region, and in general is not strictly convex. That is, the rate pairs on the line segment $|A B|$ in the figure are in general achieved by time sharing between the points $A$ and $B$. This can be shown by noting that the pentagon containing $|A B|$ corresponds to a power control policy that maximizes the sum capacity, and then proving that for correlated signature sequences, there are infinitely many rate tuples that give the same sum rate. This is stated more precisely in the following theorem.

Theorem 2: The capacity region of a power-controlled fading CDMA channel is not strictly convex, provided $\exists i, j \in\{1, \ldots, K\}$ such that $i \neq j$ and $0<\boldsymbol{s}_{i}^{\top} \boldsymbol{s}_{j}<1$.

Proof: Let $\mathcal{P}(\boldsymbol{h})=\left\{p_{1}^{*}(\boldsymbol{h}), \ldots, p_{K}^{*}(\boldsymbol{h}), \forall \boldsymbol{h}\right\}$ be a power control policy that maximizes the sum of rates of all users, i.e., the sum capacity. The capacity region corresponding to this particular power con-

$$
\underset{\left\{\boldsymbol{p}(\boldsymbol{h}): E_{\boldsymbol{h}}\left[p_{i}(\boldsymbol{h})\right] \leq \bar{p}_{i}, \forall i\right\}}{\bigcup_{i \in \Gamma}}\left\{\boldsymbol{R} R_{i} \leq E_{\boldsymbol{h}}\left[\frac{1}{2} \log \left|\boldsymbol{I}_{N}+\sigma^{-2} \sum_{i \in \Gamma} h_{i} p_{i}(\boldsymbol{h}) \boldsymbol{s}_{i} \boldsymbol{s}_{i}^{\top}\right|\right]\right\}, \quad \forall \Gamma \subset\{1, \ldots, K\} .
$$


trol policy is a polymatroid $\mathcal{G}_{\mathcal{P}(\boldsymbol{h})}$, with corners in the positive "quadrant" given by

$$
\begin{array}{r}
R_{\boldsymbol{\Gamma}(k+1)}=E_{\boldsymbol{h}}\left[\frac{1}{2} \log \frac{\left|\boldsymbol{I}_{N}+\sigma^{-2} \boldsymbol{S}_{\Gamma_{k+1}} \boldsymbol{D}_{\Gamma_{k+1}}(\boldsymbol{h}) \boldsymbol{S}_{\Gamma_{k+1}}^{\top}\right|}{\left|\boldsymbol{I}_{N}+\sigma^{-2} \boldsymbol{S}_{\Gamma_{k}} \boldsymbol{D}_{\Gamma_{k}}(\boldsymbol{h}) \boldsymbol{S}_{\Gamma_{k}}^{\top}\right|}\right], \\
k=0, \ldots, K-1
\end{array}
$$

where $\boldsymbol{S}=\left[\boldsymbol{s}_{1} \ldots \boldsymbol{s}_{K}\right], \boldsymbol{D}(\boldsymbol{h})=\operatorname{diag}\left[p_{1}^{*}(\boldsymbol{h}) h_{1}, \ldots, p_{K}^{*}(\boldsymbol{h}) h_{K}\right]$, $\Gamma \triangleq[\Gamma(1), \ldots, \Gamma(K)]$ is any permutation of $\{1, \ldots, K\}$, $\Gamma_{k} \triangleq[\Gamma(1), \ldots, \Gamma(k)]$ for $k=1, \ldots, K$, and $\Gamma_{0} \triangleq \varnothing . D_{\Gamma_{k}}$ and $\boldsymbol{S}_{\Gamma_{k}}$ refer to submatrices containing only the received powers and signature sequences of the users in the subset $\Gamma_{k}$. Each one of the $K$ ! possible permutations correspond to a corner point of the polymatroid $\mathcal{G}_{\mathcal{P}(\boldsymbol{h})}$ in the positive orthant, and these points are also the corners of the $(K-1)$-dimensional dominant face [18] of $\mathcal{G}_{\mathcal{P}(\boldsymbol{h})}$. Note that, since any point on the dominant face of $\mathcal{G}_{\mathcal{P}(\boldsymbol{h})}$ achieves the maximum sum capacity, it should also lie on the surface of the overall capacity region $\mathcal{C}$. That is, the dominant face of $\mathcal{G}_{\mathcal{P}(\boldsymbol{h})}$ constitutes a portion of the surface of $\mathcal{C}$. Therefore, for the surface of $\mathcal{C}$ to be strictly convex, we need all the corners (3) of the dominant face to collapse to a single point. It is easy to see that this condition can be summarized by

$$
\begin{array}{r}
E_{\boldsymbol{h}}\left[\log \left|\boldsymbol{I}_{N}+\sigma^{-2} \boldsymbol{S}_{E} \boldsymbol{D}_{E}(\boldsymbol{h}) \boldsymbol{S}_{E}^{\top}\right|\right]=\sum_{i \in E} E_{\boldsymbol{h}}\left[\log \left(1+\sigma^{-2} p_{i}^{*}(\boldsymbol{h}) h_{i}\right)\right], \\
\forall E \subset\{1, \ldots, K\}
\end{array}
$$

Define $\boldsymbol{Q}_{E}(\boldsymbol{h}) \triangleq \boldsymbol{S}_{E} \boldsymbol{D}_{E}(\boldsymbol{h})^{1 / 2}$. Then for all $\boldsymbol{h}$, we have

$$
\begin{aligned}
\log \left|\boldsymbol{I}_{N}+\sigma^{-2} \boldsymbol{S}_{E} \boldsymbol{D}_{E}(\boldsymbol{h}) \boldsymbol{S}_{E}^{\top}\right| & =\log \left|\boldsymbol{I}_{N}+\sigma^{-2} \boldsymbol{Q}_{E}(\boldsymbol{h}) \boldsymbol{Q}_{E}(\boldsymbol{h})^{\top}\right| \\
& =\log \left|\boldsymbol{I}_{|E|}+\sigma^{-2} \boldsymbol{Q}_{E}(\boldsymbol{h})^{\top} \boldsymbol{Q}_{E}(\boldsymbol{h})\right| \\
& \leq \sum_{i \in E} \log \left(1+\sigma^{-2} p_{i}^{*}(\boldsymbol{h}) h_{i}\right)
\end{aligned}
$$

where the last step follows from Hadamard's inequality [8], and the equality is achieved if and only if $\boldsymbol{W}_{E}(\boldsymbol{h}) \triangleq Q_{E}(\boldsymbol{h})^{\top} \boldsymbol{Q}_{E}(\boldsymbol{h})$ is diagonal. Since (7) holds for all $\boldsymbol{h}$, (4) holds when and only when $\boldsymbol{W}_{E}(\boldsymbol{h})$ is diagonal for almost all $h$ (i.e., with probability 1 ). For equality in (7), we need

$$
\left[\boldsymbol{Q}_{E}(\boldsymbol{h})^{\top} \boldsymbol{Q}_{E}(\boldsymbol{h})\right]_{i, j}=\sqrt{p_{i}^{*}(\boldsymbol{h}) p_{j}^{*}(\boldsymbol{h}) h_{i} h_{j}} \boldsymbol{s}_{i} \boldsymbol{s}_{j}=0, \quad \forall i \neq j
$$

or equivalently

$$
p_{i}^{*}(\boldsymbol{h}) p_{j}^{*}(\boldsymbol{h})=0 \quad \vee \quad \boldsymbol{s}_{i}^{\top} \boldsymbol{s}_{j}=0, \quad \forall i \neq j, \forall \boldsymbol{h} .
$$

Note that this condition is readily satisfied if $K \leq N$ and the signature sequences of all users are orthogonal, in which case the sum rate is achieved at a single point rather than on a hyperplane (i.e., on the dominant face of the corresponding polymatroid). Therefore, let us focus on nonorthogonal sequences. Let $\boldsymbol{s}_{i}^{\top} \boldsymbol{s}_{j} \neq 0$ for $i \neq j$. Then, for strict convexity of $\mathcal{C}$, we need $p_{i}^{*}(\boldsymbol{h}) p_{j}^{*}(\boldsymbol{h})=0$ for almost all $\boldsymbol{h}$, i.e., except over a zero probability subset of channel states. In other words, the optimal power allocation policy which achieves the sum capacity should dictate no more than one user transmit simultaneously with nonzero probability. In Section IV, it will be shown that this is true if and only if the signature sequences of all users are identical, which establishes that $\mathcal{C}$ is not strictly convex unless all signature sequences are identical or orthogonal.

In proving Theorem 2, we made use of the properties of sum-capacity-achieving power allocation policy. Sum capacity is often considered as a figure of merit for multiuser systems, because of the ease with which it can be handled as an objective function, as opposed to the more difficult to handle arbitrary rate tuples on the boundary of the capacity region. In Section III-B, we give the optimal power allocation policy that maximizes the sum capacity, and propose an algorithm that updates the powers of the users iteratively and converges to this policy. In Section IV, we investigate some properties of this optimal power allocation policy.

\section{B. Sum Capacity, and Optimal Power Allocation via Iterative Waterfilling}

For the CDMA system given by (1), we would like to characterize the optimum power allocation policy which maximizes the ergodic sum capacity, i.e., which is the solution to

$$
\begin{aligned}
\max _{\left\{p_{i}(\boldsymbol{h})\right\}} & \frac{1}{2} \int \log \left|\boldsymbol{I}_{N}+\sigma^{-2} \sum_{i=1}^{K} h_{i} p_{i}(\boldsymbol{h}) \boldsymbol{s}_{i} \boldsymbol{s}_{i}^{\top}\right| f(\boldsymbol{h}) d \boldsymbol{h} \\
\text { s.t. } & \int p_{i}(\boldsymbol{h}) f(\boldsymbol{h}) d \boldsymbol{h}=\bar{p}_{i}, \quad p_{i}(\boldsymbol{h}) \geq 0, \quad i=1, \ldots, K
\end{aligned}
$$

where $f(\boldsymbol{h})$ is the joint probability density function (pdf) of the channel states. For arbitrary signature sequences, no closed-form solution for this problem is known. It is interesting to note that (10) reduces to the Knopp-Humblet problem [10] if the signature sequences are identical, i.e., $\boldsymbol{s}_{i}=\boldsymbol{s}$ for all $i$, and it reduces to $K$ separable Goldsmith-Varaiya [7] problems, if the signature sequences are orthogonal, i.e., $\boldsymbol{s}_{i}^{\top} \boldsymbol{s}_{j}=0$ for $i \neq j$, in which case each problem can be solved independently of the others. Our aim is to characterize the optimal power allocation policy for the most general case where the signature sequences are arbitrarily correlated, i.e., $\boldsymbol{s}_{i}^{\top} \boldsymbol{s}_{j}$ is not restricted to be zero or one, and investigate the properties of this policy.

We can express the ergodic sum capacity, the objective function of (10), as

$$
C_{\mathrm{sum}}=C_{k}+\bar{C}_{k}
$$

where

$$
C_{k}=\frac{1}{2} \int \log \left(1+h_{k} p_{k}(\boldsymbol{h}) \boldsymbol{s}_{k}^{\top} \boldsymbol{A}_{k}^{-1} \boldsymbol{s}_{k}\right) f(\boldsymbol{h}) d \boldsymbol{h}
$$

represents the contribution of the $k$ th user to the sum capacity when the transmit powers of all other users at all channel states are fixed, and $\bar{C}_{k}$ represents the sum capacity of the remaining users when the $k$ th user is removed from the system. In (12), $\boldsymbol{A}_{k}$ is defined as

$$
\boldsymbol{A}_{k}=\sigma^{2} \boldsymbol{I}_{N}+\sum_{i \neq k} h_{i} p_{i}(h) \boldsymbol{s}_{i} \boldsymbol{s}_{i}^{\top}
$$

It is worth noting that $C_{\text {sum }}$, the objective function in (10), is a concave function of the powers, and moreover, provided that the matrices $\left\{\boldsymbol{s}_{i} \boldsymbol{s}_{i}^{\top}\right\}_{i=1}^{K}$ are linearly independent, it is a strictly concave function of the powers [13, Proposition 4.2]. Also, the constraint set in (10) is convex. Therefore, the optimization problem in (10) has a unique global optimum when $\left\{\boldsymbol{s}_{i} \boldsymbol{s}_{i}^{\top}\right\}_{i=1}^{K}$ are linearly independent; and all local optimums yield the same objective function value otherwise. A more general version of this optimization problem with multiple antennas is solved in [12], where the solution is left in terms of the Karush-Kuhn-Tucker (KKT) conditions. Here, we derive the solution of the power control problem specifically for the CDMA system, as we shall use it in our future discussions in Section IV. The extended KKT conditions with mixed constraints [19, Ch. 13] reduce to

$$
\frac{h_{k} \boldsymbol{s}_{k}^{\top} \boldsymbol{A}_{k}^{-1} \boldsymbol{s}_{k}}{1+p_{k}(\boldsymbol{h}) h_{k} \boldsymbol{s}_{k}^{\top} \boldsymbol{A}_{k}^{-1} \boldsymbol{s}_{k}} \leq \lambda_{k}, \quad k=1, \ldots, K, \quad \forall \boldsymbol{h} \in R^{K}
$$


which is satisfied with equality if $p_{k}>0$. Using the fact that $p_{k} \geq 0$ for all $k,(14)$ implies that the capacity-maximizing power allocation policy satisfies

$$
p_{k}(\boldsymbol{h})=\left(\frac{1}{\lambda_{k}}-\frac{1}{h_{k} \boldsymbol{s}_{k}^{\top} \boldsymbol{A}_{k}^{-1} \boldsymbol{s}_{k}}\right)^{+}, \quad k=1, \ldots, K
$$

for any realization of the channel $\boldsymbol{h}$. Here, the Lagrange multipliers $\lambda_{k}$ are determined by inserting (15) into the average power constraints in (10). The values of $\lambda_{k}$ 's depend on the statistical characterization of the channel and the choice of signature sequences. This solution is similar in structure to the solution in [13], however, it is more general in that it is valid for any continuous joint fading distribution, any power constraints, and any finite number of deterministic signature sequences, as opposed to the symmetric and asymptotical situation in [13]. Note that even though the continuity and independence assumptions on fading will be needed in order to prove the simultaneous transmission conditions for the optimal power allocation policy in Section IV, the characterization of optimal power allocation policy in (15) does not require these assumptions.

For arbitrary signature sequences, the set of equation (15) is highly nonlinear, and their solution is intractable for systems with large numbers of users. Note that (15) implies that all users should simultaneously waterfill on the "base levels" constituted by the inverse of the SIRs they would obtain if they transmitted with unit powers, i.e., $h_{k} \boldsymbol{s}_{k}^{\top} \boldsymbol{A}_{k}^{-1} \boldsymbol{s}_{k}$ for user $k$. Since solving for the simultaneous waterfilling (where each user's power allocation is given by a single-user waterfilling, but depends on the other users' powers) solution for all users seems intractable, we devise an iterative algorithm. Consider optimizing for the power of only user $k$ over all channel states, given the powers of all other users at all channel states

$$
\begin{aligned}
p_{k}^{n+1} & =\arg \max _{p_{k}} C_{\text {sum }}\left(p_{1}^{n+1}, \ldots, p_{k-1}^{n+1}, p_{k}, p_{k+1}^{n} \ldots, p_{K}^{n}\right) \\
& =\arg \max _{p_{k}} C_{k}\left(p_{k}\right)
\end{aligned}
$$

where $C_{k}\left(p_{k}\right)$ denotes the contribution of user $k$ to $C_{\text {sum }}$, as defined in (12). $C_{k}\left(p_{k}\right)$ depends on the power distributions and signature sequences of all other users through $\boldsymbol{A}_{k}$ 's which change as a function of the channel state. The solution of (16) can be found as a single-user waterfilling over all channel states of the system,

$$
p_{k}(\boldsymbol{h})=\left(\frac{1}{\tilde{\lambda}_{k}}-\frac{1}{h_{k} \boldsymbol{s}_{k}^{\top} \boldsymbol{A}_{k}^{-1} \boldsymbol{s}_{k}}\right)^{+}
$$

where $\tilde{\lambda}_{k}$ is the Lagrange multiplier corresponding to the single-user optimization problem in (16). If we let only one user allocate its power over all channel states using (17), and iterate over all users sequentially, this iterative one-user-at-a-time algorithm is guaranteed to converge to the global optimum solution of (10), since the objective function $C_{\text {sum }}$ is a concave function of powers, $C_{k}\left(p_{k}\right)$ given by (12) is a strictly concave function of $p_{k}$, the constraint set for powers over which the maximization is to be performed is convex, and has a Cartesian product structure among the users, see [20, Proposition 3.9].

\section{Properties of the Optimal Power Allocation}

Let us now consider the inverse problem of finding the channel state of the system for a given transmit power vector with nonzero components. Since all components of the power vector are nonzero, this means that all users transmit simultaneously at this particular channel state, and (14) should be satisfied with equality for all $k$. Therefore, given any arbitrary power vector $p$ with $0<p_{i}<1 / \lambda_{i}$, the channel state where this power vector is used can be found by solving

$$
\boldsymbol{h}=\boldsymbol{f}(\boldsymbol{h})
$$

where the vector function $\boldsymbol{f}(\boldsymbol{h})$ is defined as

$$
f_{k}(\boldsymbol{h})=\frac{\lambda_{k}}{\left(1-\lambda_{k} p_{k}\right)} \frac{1}{\boldsymbol{s}_{k}^{\top} \boldsymbol{A}_{k}^{-1} \boldsymbol{s}_{k}}, \quad k=1, \ldots, K .
$$

Our first goal is to show that there exists a unique vector $\boldsymbol{h}$ of channel states corresponding to any given nonzero solution $p$ to the power control problem. To this end, we first need to prove some properties of the function $f(\boldsymbol{h})$.

Definition 1 ([5]): $\boldsymbol{f}(\boldsymbol{h})$ is standard, if for all $\boldsymbol{h} \geq \mathbf{0}$, the following properties are satisfied.

- Positivity: $\boldsymbol{f}(\boldsymbol{h})>0$.

- Monotonicity: If $\boldsymbol{h} \geq \boldsymbol{h}^{\prime}$ then $\boldsymbol{f}(\boldsymbol{h}) \geq \boldsymbol{f}\left(\boldsymbol{h}^{\prime}\right)$.

- Scalability: For all $\alpha>1, \alpha \boldsymbol{f}(\boldsymbol{h})>\boldsymbol{f}(\alpha \boldsymbol{h})$

Lemma 1: Let $0<p_{k}<1 / \lambda_{k}$, for all $k$. Then, $\boldsymbol{f}(\boldsymbol{h})$ is standard. Proof: For notational convenience, let us define

$$
\begin{aligned}
g_{k}\left(\boldsymbol{h}, \boldsymbol{c}_{k}\right) & =\frac{\lambda_{k}}{\left(1-\lambda_{k} p_{k}\right)} \frac{\sum_{i \neq k} p_{i} h_{i}\left(\boldsymbol{c}_{k}^{\top} \boldsymbol{s}_{i}\right)^{2}+\sigma^{2}\left(\boldsymbol{c}_{k}^{\top} \boldsymbol{c}_{k}\right)}{\left(\boldsymbol{c}_{k}^{\top} \boldsymbol{s}_{k}\right)^{2}} \\
& =\frac{\lambda_{k}}{\left(1-\lambda_{k} p_{k}\right)} \frac{\boldsymbol{c}_{k}^{\top} \boldsymbol{A}_{k} \boldsymbol{c}_{k}}{\left(\boldsymbol{c}_{k}^{\top} \boldsymbol{s}_{k}\right)^{2}} .
\end{aligned}
$$

Then, interpreting $c_{k}$ as a linear receiver filter, we can relate $f_{k}(\boldsymbol{h})$ to $g_{k}\left(\boldsymbol{h}, \boldsymbol{c}_{k}\right)$ by

$$
f_{k}(\boldsymbol{h})=\min _{\boldsymbol{c}_{k}} g_{k}\left(\boldsymbol{h}, c_{k}\right)
$$

where the filter that minimizes $g_{k}\left(\boldsymbol{h}, \boldsymbol{c}_{k}\right)$ is $\boldsymbol{c}_{k}^{*}=\boldsymbol{A}_{k}^{-1} \boldsymbol{s}_{k}$, i.e., a scaled version of the well-known MMSE filter.

For $0<p_{k}<1 / \lambda_{k}, g_{k}\left(\boldsymbol{h}, \boldsymbol{c}_{k}\right)>0$ for any $\boldsymbol{c}_{k}$, due to nonzero noise variance. Then, $f_{k}(\boldsymbol{h})=\min _{\boldsymbol{c}_{k}} g_{k}\left(\boldsymbol{h}, \boldsymbol{c}_{k}\right)>0$ proving the positivity.

For monotonicity, let $\boldsymbol{h} \geq \boldsymbol{h}^{\prime}$

$$
\begin{aligned}
f_{k}(\boldsymbol{h}) & =\min _{\boldsymbol{c}_{k}} g_{k}\left(\boldsymbol{h}, \boldsymbol{c}_{k}\right) \\
& =g_{k}\left(\boldsymbol{h}, \boldsymbol{c}_{k}^{*}\right) \\
& \geq g_{k}\left(\boldsymbol{h}^{\prime}, \boldsymbol{c}_{k}^{*}\right) \\
& \geq \min _{\boldsymbol{c}_{k}} g_{k}\left(\boldsymbol{h}^{\prime}, \boldsymbol{c}_{k}\right)=f_{k}\left(\boldsymbol{h}^{\prime}\right) .
\end{aligned}
$$

Inequality (25) follows from (20) noting that $\boldsymbol{h} \geq \boldsymbol{h}^{\prime}$ and $\boldsymbol{c}_{k}$ is fixed.

For scalability, we pick $\alpha>1$

$$
\begin{aligned}
\alpha f_{k}(\boldsymbol{h}) & =\alpha \min _{\boldsymbol{c}_{k}} g_{k}\left(\boldsymbol{h}, \boldsymbol{c}_{k}\right) \\
& =\alpha g_{k}\left(\boldsymbol{h}, \boldsymbol{c}_{k}^{*}\right) \\
& >g_{k}\left(\alpha \boldsymbol{h}, \boldsymbol{c}_{k}^{*}\right) \\
& \geq \min _{\boldsymbol{c}_{k}} g_{k}\left(\alpha \boldsymbol{h}, \boldsymbol{c}_{k}\right)=f_{k}(\alpha \boldsymbol{h}) .
\end{aligned}
$$

Inequality (29) follows from (20) noting that $\alpha>1$ and $\boldsymbol{c}_{k}$ is fixed.

Note that, since $\boldsymbol{f}(\boldsymbol{h})$ is standard, if there is a solution for (18), it is unique [5]. In fact, one can devise an iterative algorithm to find this solution

$$
\boldsymbol{h}(n+1)=\boldsymbol{f}(\boldsymbol{h}(n)) .
$$

It is interesting to note that the problem in (18) with the definition of $\boldsymbol{f}(\boldsymbol{h})$ in (19) is very similar to the joint power control and linear receiver filter design problem studied in [21].

In [21], the problem is to solve for the componentwise smallest power vector $\boldsymbol{p}$ and the receiver filters $\left\{\boldsymbol{c}_{i}\right\}_{i=1}^{K}$ such that all users satisfy their SIR-based quality-of-service requirements. For a single receiver site (e.g., single-cell) system, the problem becomes that of 
finding componentwise smallest power vector and receiver filters that satisfy

$$
\operatorname{SIR}_{k}=\frac{p_{k} h_{k}\left(\boldsymbol{c}_{k}^{\top} \boldsymbol{s}_{k}\right)^{2}}{\sum_{i \neq k} p_{i} h_{i}\left(\boldsymbol{c}_{k}^{\top} \boldsymbol{s}_{i}\right)^{2}+\sigma^{2}\left(\boldsymbol{c}_{k}^{\top} \boldsymbol{c}_{k}\right)} \geq \beta_{k}
$$

where $\beta_{k}, k=1, \ldots, K$ are the SIR targets.

When there are no maximum power constraints, solving for optimum transmit powers $\boldsymbol{p}$ and received powers $\boldsymbol{q}$ where $q_{k}=p_{k} h_{k}$ are equivalent. The optimum transmit powers can be found using the optimum received powers via $p_{k}^{*}=q_{k}^{*} / h_{k}$. Then, from (32) and (13)

$$
\operatorname{SIR}_{k}=\frac{q_{k}\left(\boldsymbol{c}_{k}^{\top} \boldsymbol{s}_{k}\right)^{2}}{\boldsymbol{c}_{k}^{\top} \boldsymbol{A}_{k} \boldsymbol{c}_{k}} \geq \beta_{k} .
$$

For any given powers, $c_{k}$ should be chosen to be the MMSE filter as it maximizes the SIR [21]. Using the MMSE filters $\boldsymbol{c}_{k}=\alpha_{k} \boldsymbol{A}_{k}^{-1} \boldsymbol{s}_{k}$, the problem becomes that of solving for $\boldsymbol{q}$ in

$$
q_{k} \boldsymbol{s}_{k}^{\top} \boldsymbol{A}_{k}^{-1} \boldsymbol{s}_{k}=\beta_{k} \text {. }
$$

While [21] developed a distributed iterative algorithm that converges to the optimum powers (and receivers) assuming that the problem is feasible, [22] found the conditions on the SIR targets $\left\{\beta_{i}\right\}_{i=1}^{K}$ and the signature sequences $\left\{\boldsymbol{s}_{i}\right\}_{i=1}^{K}$ that guarantee that the problem is feasible, i.e., positive $q_{k}$ 's that satisfy (34) exist. The SIR targets $\beta_{1}, \ldots, \beta_{k}$ are feasible if and only if [22, Theorem 10]

$$
\sum_{k \in U} \frac{\beta_{k}}{1+\beta_{k}}<\operatorname{rank}(\boldsymbol{S}(U)), \quad \forall U \subset\{1, \ldots, K\}
$$

where $\boldsymbol{S}(U)$ is the matrix containing the signature sequences of the users in the subset $U$.

In our problem, the channel gains are found for any given power vector by solving (18),

$$
h_{k} p_{k} \boldsymbol{s}_{k}^{\top} \boldsymbol{A}_{k}^{-1} \boldsymbol{s}_{k}=\frac{\lambda_{k} p_{k}}{\left(1-\lambda_{k} p_{k}\right)} .
$$

Since there are no maximum constraints on the channel gains, solving for $h_{k}$ and $q_{k}=h_{k} p_{k}$ are equivalent, as we can obtain the solution for $h_{k}$ using the solution for $q_{k}$ via $h_{k}^{*}=q_{k}^{*} / p_{k}$. Thus, our problem is equivalent to (34) where $\beta_{k}$ are given by

$$
\beta_{k}=\frac{\lambda_{k} p_{k}}{\left(1-\lambda_{k} p_{k}\right)}, \quad k=1, \ldots, K
$$

and are determined by the given power vector. The set of feasible powers can then be found by inserting (37) into (35)

$$
\sum_{k \in U} \lambda_{k} p_{k}<\operatorname{rank}(\boldsymbol{S}(U)), \quad \forall U \subset\{1, \ldots, K\} .
$$

Therefore, once we fix a power vector satisfying (38), (18) has a unique solution, since $\boldsymbol{f}(\boldsymbol{h})$ is standard. That is, the power vector we chose is a possible candidate for the optimum power allocation at the channel state obtained by solving (18). This means that, corresponding to a set of feasible power vectors, there always exists a set of channel states where all of the users in the system transmit simultaneously. This set, however, can have zero probability as in [10], which is the result of the fact that, although we can find a unique channel state for a feasible power vector, multiple feasible power vectors may correspond to the same channel state, i.e., there may be multiple optimum power vectors with the same $C_{\text {sum }}$. That is, the mapping between the powers and the channel states is not one-to-one, in general.

The significance of (38) for our purposes is that the set of feasible power vectors constitutes a volume in $K$-dimensional space. For the set of feasible power vectors satisfying (38), and having strictly positive components, if the set of corresponding channel states found by solving (18) have a nonzero measure, then we can conclude that all users transmit simultaneously with a positive probability.

Theorem 3: There exists a nonzero probability region of fading states $\boldsymbol{h}$ where all $K$ users transmit simultaneously, if and only if $\left\{\boldsymbol{s}_{i} \boldsymbol{s}_{i}^{\top}\right\}_{i=1}^{K}$ are linearly independent.

Proof: It is clear that the set of feasible powers as given by (38) constitutes a volume $V$ in $R^{K}$. Let us then pick any point $p_{0}>0$ in this set, and compute the channel state which corresponds to this particular solution of powers. By feasibility of $\boldsymbol{p}_{0}$, the resulting channel state $\boldsymbol{h}_{0}$ is unique, and the original vector $\boldsymbol{p}_{0}$ satisfies the KKT conditions at $\boldsymbol{h}_{0}$. Given $\left\{\boldsymbol{s}_{i} \boldsymbol{s}_{i}^{\top}\right\}_{i=1}^{K}$ are linearly independent, we know that there exists a unique global maximum for $C_{\text {sum }}$ since it is strictly concave. Therefore, the waterfilling solution we get at the fading state $\boldsymbol{h}_{0}$ should be equal to $\boldsymbol{p}_{0}$, as it is a possible solution to the problem, and the problem has a unique global optimum. Hence, we obtain a unique fading state for a power level, and a unique power for a fading state, for a set of powers satisfying (38). This implies that there exists a one-to-one mapping from the space of feasible strictly positive powers to the space of fading states. This one-to-one mapping maps the volume $V \subset R^{K}$ of feasible powers to a volume of fading states $\tilde{V} \subset R^{K}$ implying that the resulting set of fading states where $K$ users transmit simultaneously has nonzero probability. This completes the proof of the if part.

For the only if part, consider the case where $\left\{\boldsymbol{s}_{i} \boldsymbol{s}_{i}^{\top}\right\}_{i=1}^{K}$ are linearly dependent. For all $K$ users to transmit simultaneously with nonzero powers, (14) must be satisfied with equality for all $k$. By applying the matrix inversion lemma, and defining $\boldsymbol{A}=\sigma^{2} \boldsymbol{I}_{N}+\boldsymbol{S P} \boldsymbol{S}^{\top}$, which contains all users' powers and signatures, (14) can be written in the alternative form

$$
h_{k} \boldsymbol{s}_{k}^{\top} \boldsymbol{A}^{-1} \boldsymbol{s}_{k}=\lambda_{k}, \quad k=1, \ldots, K .
$$

Each of these equations can also be rewritten as

$$
h_{k} \operatorname{tr}\left(\boldsymbol{A}^{-1} \boldsymbol{s}_{k} \boldsymbol{s}_{k}^{\top}\right)=\lambda_{k}, \quad k=1, \ldots, K .
$$

If $\left\{\boldsymbol{s}_{i} \boldsymbol{s}_{i}^{\top}\right\}_{i=1}^{K}$ are linearly dependent, then any one of the elements of this set, say $\boldsymbol{s}_{k} \boldsymbol{s}_{k}^{\top}$, can be written as a linear combination of the others, say, with coefficients $\alpha_{i}$, not all equal to zero. Thus,

$$
h_{k} \operatorname{tr}\left(\boldsymbol{A}^{-1} \sum_{i \neq k} \alpha_{i} \boldsymbol{s}_{i} \boldsymbol{s}_{i}^{\top}\right)=h_{k} \sum_{i \neq k} \alpha_{i} \boldsymbol{s}_{i}^{\top} \boldsymbol{A}^{-1} \boldsymbol{s}_{i}=\lambda_{k}
$$

and using (39) in (41), we get

$$
\sum_{i \neq k} \alpha_{i} \frac{\lambda_{i}}{h_{i}}=\frac{\lambda_{k}}{h_{k}}
$$

This means that, if $\left\{\boldsymbol{s}_{i} \boldsymbol{s}_{i}^{\top}\right\}_{i=1}^{K}$ are linearly dependent, then regardless of the power levels, for all users to transmit simultaneously, the channel states should satisfy (42). Since the channel states are continuous random variables, this event has zero probability. Therefore, given that $\left\{\boldsymbol{s}_{i} \boldsymbol{s}_{i}^{\top}\right\}_{i=1}^{K}$ are linearly dependent, all $K$ users transmit simultaneously only with zero probability.

Therefore, the necessary and sufficient condition for all $K$ users to transmit simultaneously with nonzero probability is that the signature sequences are such that the matrices $\left\{\boldsymbol{s}_{i} \boldsymbol{s}_{i}^{\top}\right\}_{i=1}^{K}$ are linearly independent. Our first corollary states that if the signature sequences $\left\{\boldsymbol{s}_{i}\right\}_{i=1}^{K}$ are linearly independent, then $\left\{\boldsymbol{s}_{i} \boldsymbol{s}_{i}^{\top}\right\}_{i=1}^{K}$ are linearly independent and all users transmit simultaneously with nonzero probability.

Corollary 1: When $K \leq N$, for a set of $K$ linearly independent signature sequences, there always exists a nonzero probability region of channel states where all $K$ users transmit simultaneously. 


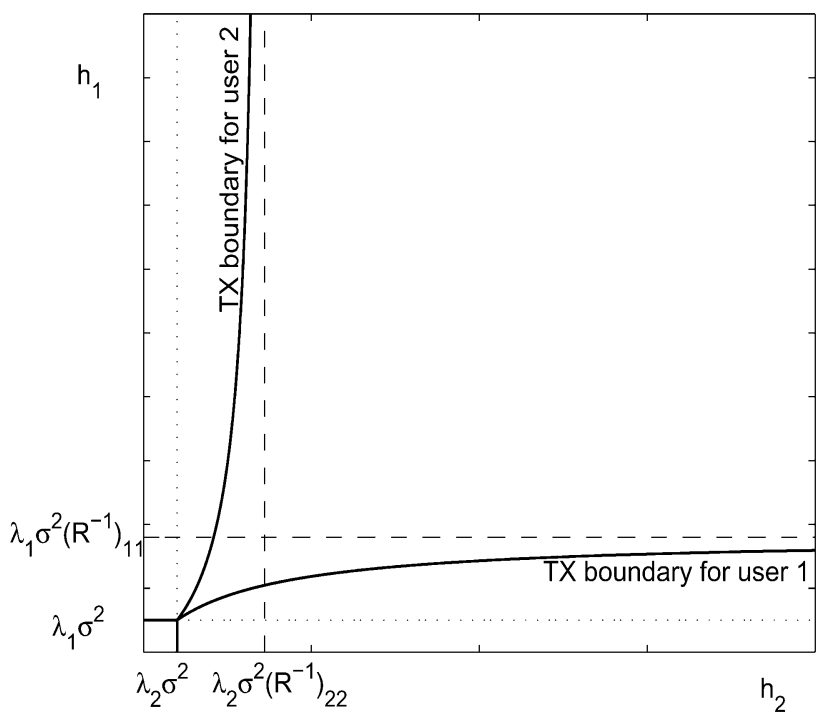

Fig. 2. Transmit region boundaries for two users with correlated signature sequences.

Proof: Assume that $\left\{\boldsymbol{s}_{i}\right\}_{i=1}^{K}$ are linearly independent. For $\left\{\boldsymbol{s}_{i} \boldsymbol{s}_{i}^{\top}\right\}_{i=1}^{K}$ to be linearly dependent, we should be able to write

$$
\boldsymbol{s}_{k} \boldsymbol{s}_{k}^{\top}=\sum_{i \neq k} \alpha_{i} \boldsymbol{s}_{i} \boldsymbol{s}_{i}^{\top}
$$

with at least two nonzero $\alpha_{i}$ 's; if only one $\alpha_{i}$ is nonzero, this implies that two signature sequences are the same violating the fact that $\left\{\boldsymbol{s}_{i}\right\}_{i=1}^{K}$ are linearly independent. The ranks of both sides of (43) have to be equal. As $\left\{\boldsymbol{s}_{i}\right\}_{i=1}^{K}$ are linearly independent, the rank of the righthand side is equal to at least two, whereas that of the left-hand side is always one. Therefore, the set $\left\{\boldsymbol{s}_{i} \boldsymbol{s}_{i}^{\top}\right\}_{i=1}^{K}$ are linearly independent for linearly independent signature sequences, and the result follows from Theorem 3.

It is hard to find closed-form expressions for the region of the channel gains where all users transmit simultaneously. For a simple two-user system, it can be shown that both users transmit with nonzero powers when $\boldsymbol{h}$ belongs to a region expressed by

$$
h_{1}>\frac{\lambda_{1} \sigma^{2} h_{2}}{h_{2}\left(1-\rho^{2}\right)+\rho^{2} \sigma^{2} \lambda_{2}}, \quad h_{2}>\frac{\lambda_{2} \sigma^{2} h_{1}}{h_{1}\left(1-\rho^{2}\right)+\rho^{2} \sigma^{2} \lambda_{1}}
$$

where $\rho=\boldsymbol{s}_{1}^{\top} \boldsymbol{s}_{2}$ denotes the cross correlation between the signature sequences of the users. This region is depicted in Fig. 2.

It is interesting to note that when $h_{2}$ goes to infinity, the lower bound on $h_{1}$ approaches the limit $\lambda_{1} \sigma^{2} /\left(1-\rho^{2}\right)$, and as $h_{1}$ goes to infinity, the lower bound on $h_{2}$ goes to $\lambda_{2} \sigma^{2} /\left(1-\rho^{2}\right)$. These are the two (horizontal and vertical) asymptotes shown in Fig. 2. For more than two users, even though the exact expressions for the boundaries of the simultaneous transmission region are nonlinear and complex, we can describe an "orthant" of the space of all channel states where all users transmit simultaneously. This orthant is a subset of the actual simultaneous transmission region.

Theorem 4: For a set of $K$ linearly independent signature sequences, the region of channel states where all users transmit simultaneously includes an "orthant" in $R^{K}$ described by

$$
h_{k}>\lambda_{k} \sigma^{2}\left(\boldsymbol{R}^{-1}\right)_{k k}, \quad k=1, \ldots, K
$$

where $\boldsymbol{R}=\boldsymbol{S}^{\top} \boldsymbol{S}$ is the correlation matrix of the signature sequences.
Proof: From (14), user $k$ transmits when its channel state $h_{k}$ satisfies

$$
h_{k}=\frac{\lambda_{k}}{\left(1-\lambda_{k} p_{k}\right)} \frac{1}{\boldsymbol{s}_{k}^{\top} \boldsymbol{A}_{k}^{-1} \boldsymbol{s}_{k}} .
$$

The transmit power of the user satisfies $0<p_{k}<1 / \lambda_{k}$. Therefore, user $k$ transmits with nonzero power if and only if

$$
h_{k}>\frac{\lambda_{k}}{\boldsymbol{s}_{k}^{\top} \boldsymbol{A}_{k}^{-1} \boldsymbol{s}_{k}} .
$$

Comparing the right-hand side of (47) with (19), it is easy to see that it is a standard function and is increasing in $p_{i} h_{i}, i \neq k$. Thus, from the monotonicity of $\lambda_{k} / \boldsymbol{s}_{k}^{\top} \boldsymbol{A}_{k}^{-1} \boldsymbol{s}_{k}$ we have

$$
\lambda_{k} \sigma^{2} \leq \frac{\lambda_{k}}{\boldsymbol{s}_{k}^{\top} \boldsymbol{A}_{k}^{-1} \boldsymbol{s}_{k}} \leq \lambda_{k} \sigma^{2}\left(\boldsymbol{R}^{-1}\right)_{k k}
$$

where the first inequality is satisfied with equality when the received powers $p_{i} h_{i}$ of all other users are zero, and the second inequality follows from the fact that the SIR of the linear MMSE detector is always larger than or equal to the SIR of the decorrelating detector. In fact, the upper bound becomes tight as $p_{i} h_{i}, i \neq k$ go to infinity for a fixed noise variance $\sigma^{2}$, as the MMSE detector converges to the decorrelator [15]. Now, if the channel gains are such that

$$
h_{k}>\lambda_{k} \sigma^{2}\left(\boldsymbol{R}^{-1}\right)_{k k}, \quad k=1, \ldots, K
$$

using (48) we get

$$
h_{k}>\lambda_{k} \sigma^{2}\left(\boldsymbol{R}^{-1}\right)_{k k} \geq \frac{\lambda_{k}}{\boldsymbol{s}_{k}^{\top} \boldsymbol{A}_{k}^{-1} \boldsymbol{s}_{k}}, \quad k=1, \ldots, K
$$

and conclude that all users transmit in the region of channel states where $h_{k}>\lambda_{k} \sigma^{2}\left(\boldsymbol{R}^{-1}\right)_{k k}, k=1, \ldots, K$.

It is worth mentioning that Theorem 4 could also have been used to prove Corollary 1 , by noting

$$
P[\text { all users transmit }] \geq P\left[\boldsymbol{h}: h_{k}>\lambda_{k} \sigma^{2}\left(\boldsymbol{R}^{-1}\right)_{k k}\right]>0
$$

Fig. 2 illustrates the statement of Theorem 4 for two users with correlated signature sequences. The orthant described in the theorem in this case is the infinite rectangle

$$
\left(\lambda_{1} \sigma^{2}\left(\boldsymbol{R}^{-1}\right)_{11}, \infty\right) \times\left(\lambda_{2} \sigma^{2}\left(\boldsymbol{R}^{-1}\right)_{22}, \infty\right) .
$$

Since, as stated by Theorem 3, for all $K$ users to transmit simultaneously $\left\{\boldsymbol{s}_{i} \boldsymbol{s}_{i}^{\top}\right\}_{i=1}^{K}$ should be linearly independent, the number of users transmitting simultaneously with nonzero powers cannot be arbitrarily large. The following corollary to Theorem 3 gives a bound on the maximum number of users that can transmit simultaneously.

Corollary 2: For a set of $K$ signature sequences and processing gain $N$, let the rank of the signature sequence matrix $S$ be $M \leq$ $\min \{K, N\}$. Then the number of users that can transmit simultaneously cannot be larger than $\min \{K, M(M+1) / 2\}$.

Proof: If $K \leq M(M+1) / 2$, the bound is trivial. Let us focus on the case $K>M(M+1) / 2$. If $\operatorname{rank}(\boldsymbol{S})=M$, the signature sequences can be written as

$$
\boldsymbol{s}_{k}=\sum_{i=1}^{M} a_{k i} \boldsymbol{v}_{i}
$$


where the $N \times 1$ vectors $\left\{\boldsymbol{v}_{i}\right\}_{i=1}^{M}$ constitute an orthonormal basis spanning the signature sequences. Then

$$
\begin{aligned}
\sum_{k=1}^{K} \alpha_{k} \boldsymbol{s}_{k} \boldsymbol{s}_{k}^{\top} & =\sum_{k=1}^{K} \sum_{i=1}^{M} \sum_{j=1}^{M} \alpha_{k} a_{k i} a_{k j} \boldsymbol{v}_{i} \boldsymbol{v}_{j}^{\top} \\
& =\sum_{i=1}^{M} \sum_{j=1}^{M} \boldsymbol{v}_{i} \boldsymbol{v}_{j}^{\top} \sum_{k=1}^{K} \alpha_{k} a_{k i} a_{k j} \\
& =\sum_{i=1}^{M} \sum_{j=1}^{M} \beta_{i j} \boldsymbol{v}_{i} \boldsymbol{v}_{j}^{\top}=\boldsymbol{V} \boldsymbol{B} \boldsymbol{V}^{\top}
\end{aligned}
$$

where $\boldsymbol{V}$ is a matrix with columns $\boldsymbol{v}_{i}$ and $\boldsymbol{B}_{i j}=\beta_{i j}$, with $\beta_{i j}$ defined by (55). Therefore, $\left\{\boldsymbol{s}_{i} \boldsymbol{s}_{i}^{\top}\right\}_{i=1}^{K}$ are linearly independent if and only if the equality $\boldsymbol{V} \boldsymbol{B} \boldsymbol{V}^{\top}=\mathbf{0}_{N \times N}$ implies $\alpha_{k}=0, k=1, \ldots, K$. Note that $\boldsymbol{V}$ is an orthonormal matrix by construction, and if $\boldsymbol{V} \boldsymbol{B} \boldsymbol{V}^{\top}=$ $\mathbf{0}_{N \times N}$ then multiplying this by $\boldsymbol{V}^{\top}$ and $\boldsymbol{V}$ from left and right we obtain $\boldsymbol{B}=\mathbf{0}_{M \times M}$. This dictates

$$
\begin{aligned}
& \sum_{k=1}^{K} \alpha_{k} a_{k i} a_{k j}=0 \quad i, j \in\{1, \ldots, M\} \\
& \sum_{k=1}^{K} \alpha_{k} \boldsymbol{a}_{k} \boldsymbol{a}_{k}^{\top}=\mathbf{0}_{M \times M}
\end{aligned}
$$

where $\boldsymbol{a}_{k}=\left[a_{k 1}, \ldots, a_{k M}\right]^{\top}$. The dimensionality of the space of $M \times M$ symmetric matrices is $M(M+1) / 2$, therefore, if $K>$ $M(M+1) / 2$, we can find $\alpha_{k}$ not all zero, such that (57) is satisfied, and $\left\{\boldsymbol{s}_{i} \boldsymbol{s}_{i}^{\top}\right\}_{i=1}^{K}$ are guaranteed to be linearly dependent, and the result follows from Theorem 3 .

So far, we have established results that relate to the simultaneous transmission of all users in the system. In order to complete the proof of Theorem 2 of Section III-A, we need a simultaneous transmission result similar to the one in Theorem 3, for any pair of users rather than all $K$ users. The following is an extension of the simultaneous transmission result given for all users by Theorem 3 to an arbitrary subset of $\{1, \ldots K\}$.

Theorem 5: The sum capacity maximizing power control policy dictates that there exists a nonzero probability region of fading states $\boldsymbol{h}$ where a subset $E \subset\{1, \ldots, K\}$ of users transmit simultaneously, if and only if $\left\{\boldsymbol{s}_{i} \boldsymbol{s}_{i}^{\top}\right\}_{i \in E}$ are linearly independent.

Proof: The only if part follows immediately from the proof of Theorem 3, by letting $\left\{\boldsymbol{s}_{i} \boldsymbol{s}_{i}^{\top}\right\}_{i \in E}$ be linearly dependent, and writing any $\boldsymbol{s}_{k} \boldsymbol{s}_{k}^{\top}, k \in E$ as a linear combination of the remaining matrices $\left\{\boldsymbol{s}_{i} \boldsymbol{s}_{i}^{\top}\right\}_{i \in E, i \neq k}$. This, together with the KKT conditions for optimality, gives the following relation between the channel gains:

$$
\sum_{i \in E, i \neq k} \alpha_{i} \frac{\lambda_{i}}{h_{i}}=\frac{\lambda_{k}}{h_{k}}
$$

which is a zero probability event by virtue of the channel states being continuous random variables. This proves the only if part.

We show the if part by proving that the probability

$$
\mathrm{P}\left\{p_{i}(\boldsymbol{h})>0, i \in E\right\}
$$

is bounded away from zero for linearly independent $\left\{\boldsymbol{s}_{i} \boldsymbol{s}_{i}^{\top}\right\}_{i \in E}$ (see (59)-(64) at the bottom of the page). Here, (59) follows from the fact that the set on the right-hand side is a subset of that on the left-hand side, (60) follows because user $j$ does not transmit regardless of the powers of other users if $h_{j} \leq \sigma^{2} \lambda_{j}$, (61) follows from (47), and (62) follows because users $j \notin E$ have zero powers, within the set in (61). Note that in (62), the powers $p_{k}(\boldsymbol{h})$, which are given by (15), actually depend only on the channel states of users in $E$. Thus, we define

$$
p_{k}\left(\boldsymbol{h}_{E}\right)=\left.p_{k}(\boldsymbol{h})\right|_{p_{j}(\boldsymbol{h})=0}, \quad k \in E, j \notin E .
$$

Then, (63) follows from independence of the channel states for different users, where the vector $\boldsymbol{h}_{E}$ is defined as the vector of channel states for users in $E$. Clearly, the second term on the right-hand side of (63) is positive.In order to prove (64), we will interpret the first term in (63) as the probability that all users transmit simultaneously in an equivalent $|E|$ user problem. To accomplish this, consider a fictitious problem, where we have only the users $k \in E$ in another CDMA system, and users $k \in E$ still employ the signature sequences $\boldsymbol{s}_{k}$. The noise variance $\sigma^{2}$ is also the same as in our original problem (1). Say we would like to maximize the sum capacity for the new system with $|E|$ users, and let each user $i \in E$ have a power constraint given by

$$
\bar{p}_{i}^{\prime}=\int p_{i}\left(\boldsymbol{h}_{E}\right) f\left(\boldsymbol{h}_{E}\right) d \boldsymbol{h}_{E}
$$

Then power allocation $\left\{p_{i}\left(\boldsymbol{h}_{E}\right)\right\}_{i \in E}$ is optimal in the sense of maximizing the sum capacity for the fictitious subproblem. Consequently, the first term in (63) is simply the probability that all users transmit with nonzero powers for this new problem, and by Theorem 3, this probability is greater than zero as long as $\left\{\boldsymbol{s}_{i} \boldsymbol{s}_{i}^{\top}\right\}_{i \in E}$ are linearly independent, which establishes the if part.

Finally, let us now return to the proof of Theorem 2, where we established that for strict convexity of the boundary of the capacity region, no

$$
\begin{aligned}
\mathrm{P}\left\{p_{i}(\boldsymbol{h})>0, i \in E\right\} & \geq \mathrm{P}\left\{p_{i}(\boldsymbol{h})>0, i \in E, p_{j}(\boldsymbol{h})=0, j \notin E\right\} \\
& \geq \mathrm{P}\left\{p_{i}(\boldsymbol{h})>0, i \in E, h_{j} \leq \sigma^{2} \lambda_{j}, j \notin E\right\} \\
& =\mathrm{P}\left\{h_{i}>\frac{\lambda_{i}}{\boldsymbol{s}_{i}^{\top} \boldsymbol{A}_{i}^{-1} \boldsymbol{s}_{i}}, i \in E, h_{j} \leq \sigma^{2} \lambda_{j}, j \notin E\right\} \\
& =\mathrm{P}\left\{h_{i}>\frac{\lambda_{i}}{\boldsymbol{s}_{i}^{\top}\left\{\boldsymbol{I}_{N}+\sigma^{-2} \sum_{k \in E, k \neq i} h_{k} p_{k}(\boldsymbol{h}) \boldsymbol{s}_{k} \boldsymbol{s}_{k}^{\top}\right\}^{-1} \boldsymbol{s}_{i}}, i \in E, h_{j} \leq \sigma^{2} \lambda_{j}, j \notin E\right\} \\
& =\mathrm{P}\left\{h_{i}>\frac{\lambda_{i}}{\boldsymbol{s}_{i}^{\top}\left\{\boldsymbol{I}_{N}+\sigma^{-2} \sum_{k \in E, k \neq i} h_{k} p_{k}\left(\boldsymbol{h}_{E}\right) \boldsymbol{s}_{k} \boldsymbol{s}_{k}^{\top}\right\}^{-1} \boldsymbol{s}_{i}}, i \in E\right\} \mathrm{P}\left\{h_{j} \leq \sigma^{2} \lambda_{j}, j \notin E\right\} \\
& >0 .
\end{aligned}
$$




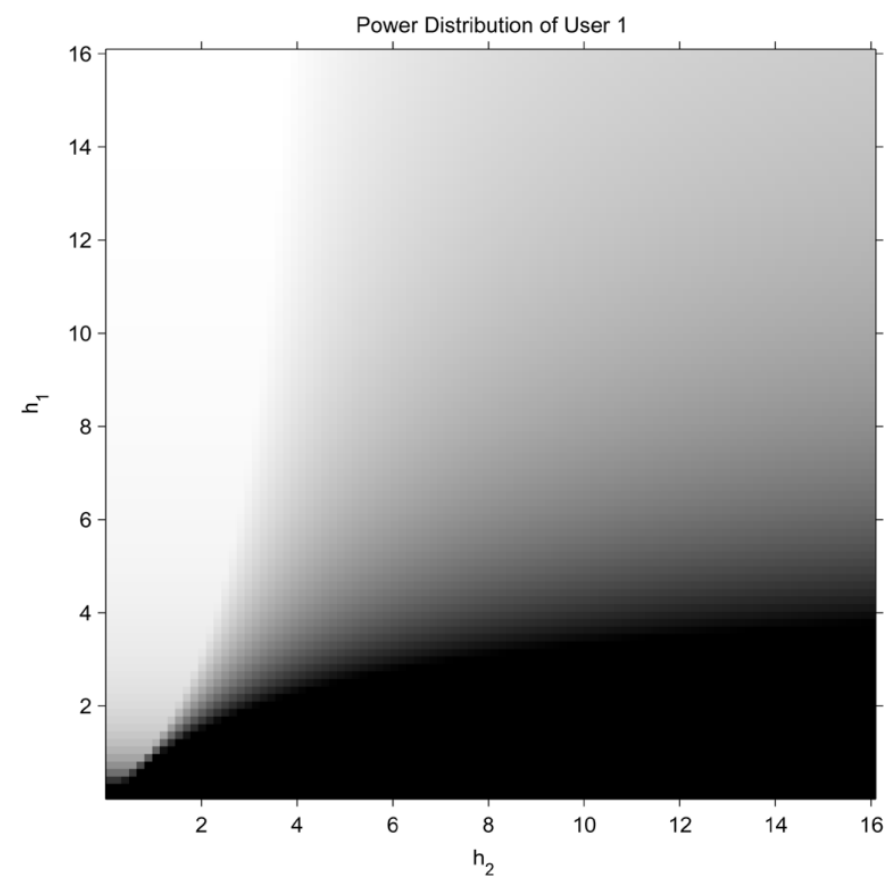

Fig. 3. Power distribution of user 1 in Rayleigh fading.

two users with nonorthogonal sequences should be transmitting simultaneously with positive probability. In the following corollary to Theorem 5, we show that two users transmit simultaneously with nonzero probability, unless they have identical signature sequences, which completes the proof of Theorem 2.

Corollary 3: Let there exist two users $i, j$ such that $0 \leq \boldsymbol{s}_{i}^{\top} \boldsymbol{s}_{j}<1$. Then, there exists a region of channel states with nonzero probability where users $i$ and $j$ transmit simultaneously.

Proof: This result follows straightforwardly from Theorem 5 by noting that $\boldsymbol{s}_{i}^{\top} \boldsymbol{s}_{j}<1$ is equivalent to two signature sequences being nonidentical, which is in turn equivalent to $\boldsymbol{s}_{i} \boldsymbol{s}_{i}^{\top}$ and $\boldsymbol{s}_{j} \boldsymbol{s}_{j}^{\top}$ being linearly independent.

\section{NUMERICAL EXAMPLES}

In this section, we give some simple numerical examples to support our analysis. Figs. 3 and 4 give an example for the two-user case where the signature sequences are correlated with $\boldsymbol{s}_{1}^{\top} \boldsymbol{s}_{2}=0.966$. In this example, the processing gain is $N=2$, the channel is an independent and identically distributed (i.i.d.) Rayleigh channel with parameter 1 , that is, $h_{k}, k=1, \ldots, K$ are exponential random variables (squares of Rayleigh random variables) with mean 1. Fig. 3 shows the power of user 1 for each fading level. In this figure, the transmit power of user 1 is represented by gray levels, lighter colors corresponding to more power. Note that, user 1 performs a single-user waterfilling wherever user 2 does not transmit. In this region, the transmit power of user 1 for a fixed $h_{1}$ is constant (independent of $h_{2}$ ). However, once user 2 starts transmitting, the "base level of the water tank" is increased, decreasing the power level of user 1 with increasing $h_{2}$. Fig. 4 shows the transmit regions in the space of channel states of the two users. The small dark region near the origin corresponds to the channel states where both users have zero power. Gray regions marked by "user 1" and "user 2" show the channel states where only one of the users transmits, whereas the white region shows the simultaneous transmit region. The simulated system corresponds to the setting in Corollary 1, and Theorem 4.

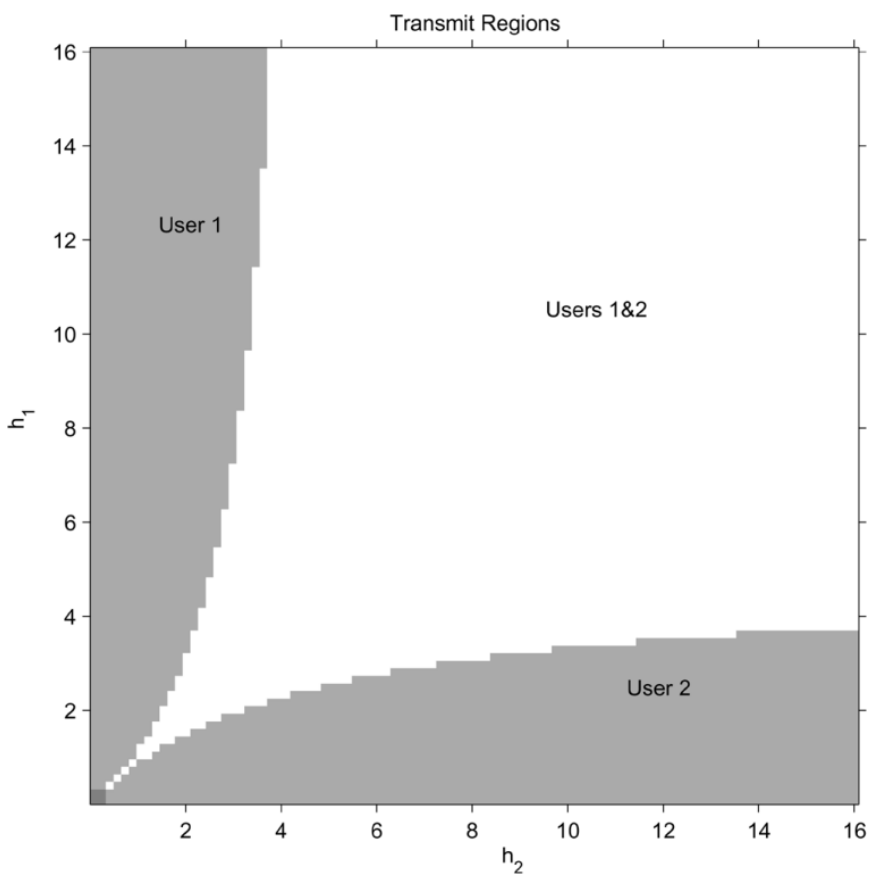

Fig. 4. Transmit regions for Rayleigh fading.

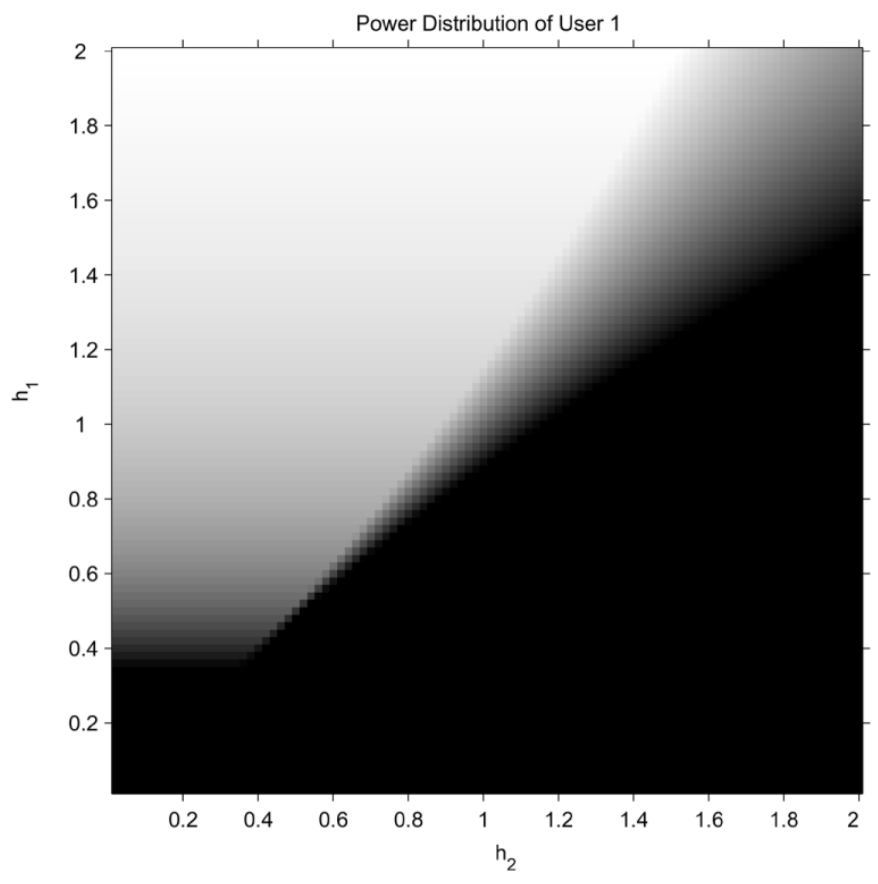

Fig. 5. Power distribution of user 1 in uniform fading.

We have noted earlier that the optimal power allocation depends on the fading distribution only through the thresholds $\lambda_{k}$. Therefore, the choice of channel fading distribution should not affect the structure of the transmit regions, except for possible shifts and scalings. To show this, we repeat our simulations for a channel where $h_{k}$ are uniform i.i.d. random variables in $(0,2]$, all other parameters being the same. Figs. 5 and 6 show the corresponding power levels and transmit regions, for this narrower span of possible channel states to emphasize better all four of the transmit regions. We see that the $\lambda_{k}$ value is slightly changed by the change in channel distribution, but the transmit regions and power distribution are very similar to the previous case. 


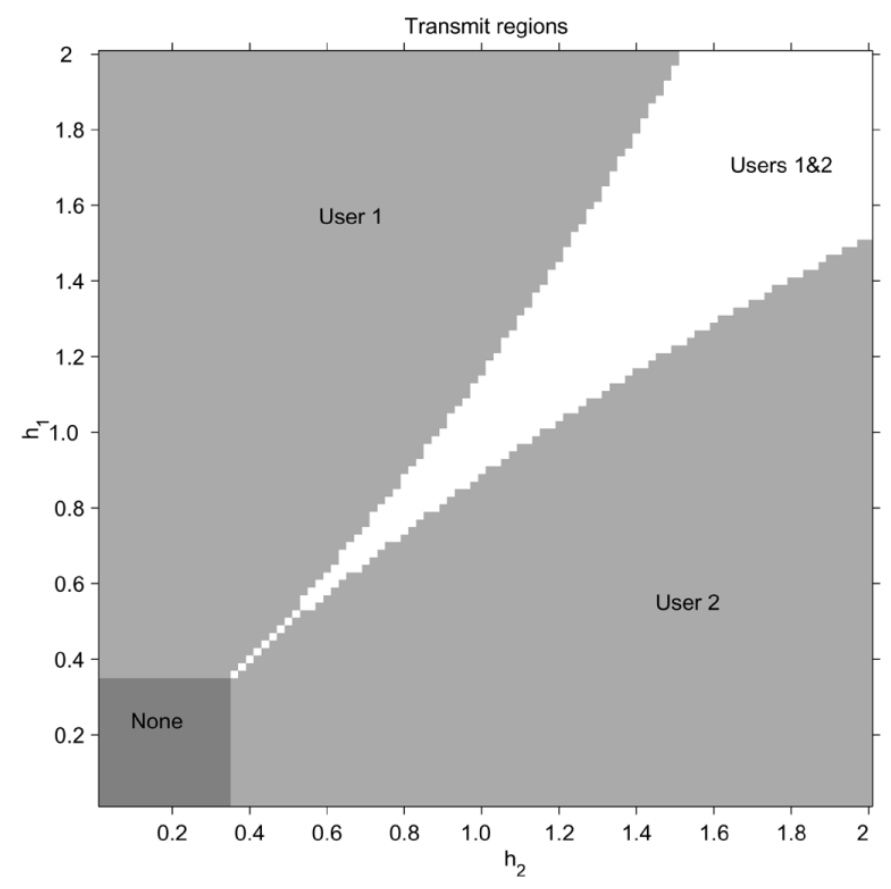

Fig. 6. Transmit regions for uniform fading.

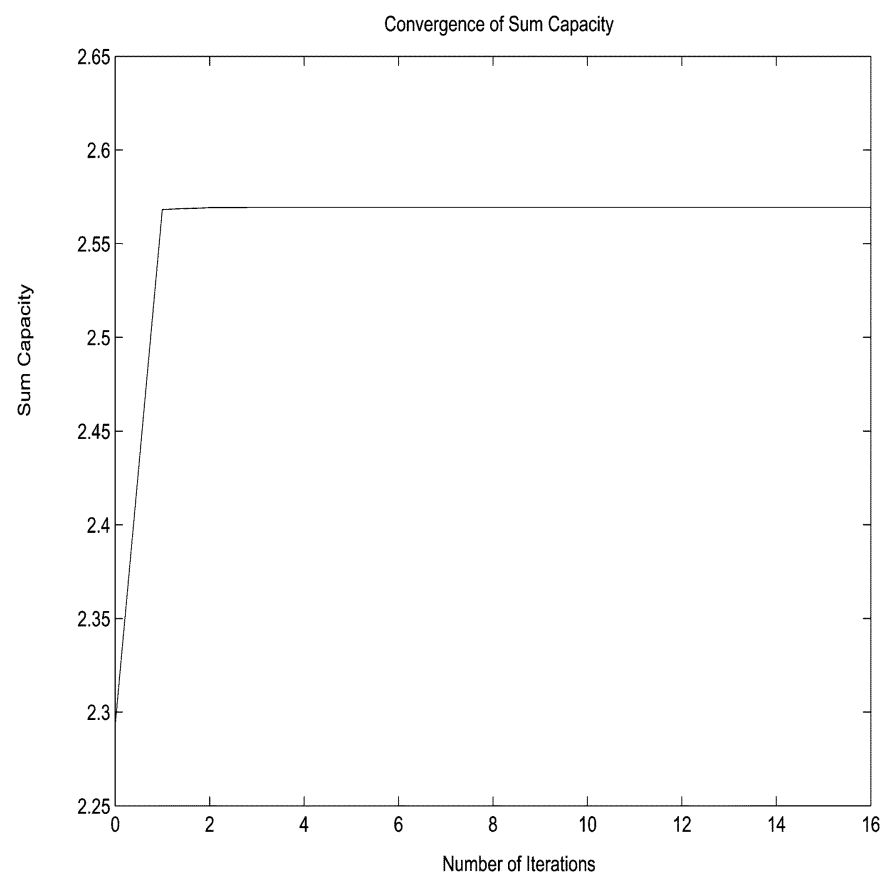

Fig. 7. Sum capacity versus number of iterations.

Fig. 7 illustrates the convergence of the iterative waterfilling algorithm to the maximum sum capacity of the system under uniform fading $\mathrm{U}(0,2]$, with average transmit powers equal to $\bar{p}_{k}=1$ and noise variance equal to $\sigma^{2}=0.1$; the convergence is quite fast as suggested by the plot.

A consequence of Theorem 3 is that we can have multiple users transmit simultaneously with nonzero probability, even when the signature sequences are linearly dependent, as long as we can have the linear independence of $\left\{\boldsymbol{s}_{i} \boldsymbol{s}_{i}^{\top}\right\}_{i=1}^{K}$. Fig. 8 shows the region where all users transmit for $K=3$ and $N=2$, the portions marked in gray correspond to the states where all three users transmit simultaneously, in the three-dimensional channel state space.

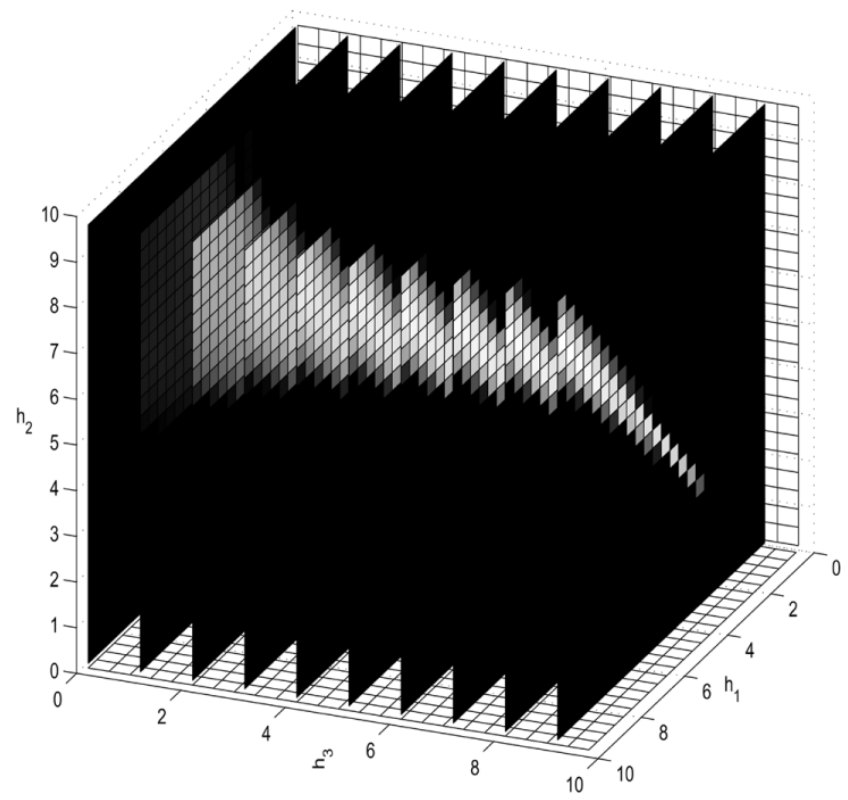

Fig. 8. Transmit region for all three users when $K=3$ and $N=2$.

In general, the probability that all users transmit simultaneously, i.e., the probability of the gray colored region, depends on the cross correlations between the signature sequences, fading statistics, and power constraints. As an example, for a system with $K=3, N=2, \bar{p}=1$, $\sigma^{2}=0.1$, uniform $\mathrm{U}(0,1)$ fading, and the correlations between the sequences $\rho_{12}=0.898, \rho_{13}=0.645, \rho_{23}=0.916$, the probability that all users transmit simultaneously is 0.245 .

\section{CONCLUSION}

We provided the capacity region for a power-controlled fading CDMA system, and proved that unless all users have orthogonal or identical sequences, it has a flat portion on which the sum capacity is maximized; i.e., it is not strictly convex. This yields the important result that, sum capacity may be achieved by infinitely many rate tuples, so one might have flexibility in choosing the individual rates of the users while keeping the sum capacity constant at its maximum.

We devised an algorithm to compute the optimum transmit powers of the users that maximize the sum capacity of a CDMA system with arbitrary signature sequences in a fading channel. The algorithm is an iterative waterfilling of powers of all users over all fading states treating at each step all other users' signals as additional colored noise. We showed that this iterative strategy converges to a globally optimum solution, and that the global optimum is unique if the signature sequence set is such that the matrices $\left\{\boldsymbol{s}_{i} \boldsymbol{s}_{i}^{\top}\right\}_{i=1}^{K}$ are linearly independent.

We also showed that the optimum power allocation scheme in the vector multiple-access channel of interest dictates more than one user to transmit simultaneously at some channel states, and the set of such channel states has a nonzero probability under certain mild conditions on the signature sequences. In fact, all $K$ users in the system are shown to transmit simultaneously with nonzero probability, if and only if $\left\{\boldsymbol{s}_{i} \boldsymbol{s}_{i}^{\top}\right\}_{i=1}^{K}$ are linearly independent. An immediate implication of this is that, if the signature sequences $\left\{\boldsymbol{s}_{i}\right\}_{i=1}^{K}$ are linearly independent, then all users transmit simultaneously in a nonzero probability region of the channel states. We extended this simultaneous transmit condition for all users to one for an arbitrary subset of users, and used it to prove the nonstrict convexity of the capacity region. We further showed that if the signature sequence matrix $\boldsymbol{S}$ of the users in the system has rank $M$, the number of users transmitting simultaneously with nonzero probability cannot be larger than $\min \{K, M(M+1) / 2\}$. 


\section{REFERENCES}

[1] O. Kaya and S. Ulukus, "Optimum power control for fading CDMA with deterministic sequences," in Proc. 40th Annu. Allerton Conf. Communications, Control and Computing, Monticello, IL, Oct. 2002, pp. 397-406.

[2] — "Capacity region of power controlled fading CDMA: Transmit strategies and convexity issues," in Proc. IEEE Int. Symp. Information Theory, Chicago, IL, June/July 2004, p. 353.

[3] J. Zander, "Performance of optimum transmitter power control in cellular radio systems," IEEE Trans. Veh. Technol., vol. 41, pp. 57-62, Feb. 1992.

[4] G. J. Foschini and Z. Miljanic, "A simple distributed autonomous power control algorithm and its convergence," IEEE Trans. Veh. Technol., vol. 42, pp. 641-646, Nov. 1993.

[5] R. D. Yates, "A framework for uplink power control in cellular radio systems,” IEEE J. Select. Areas Commun., vol. 13, pp. 1341-1347, Sept. 1995.

[6] S. V. Hanly, "An algorithm of combined cell-site selection and power control to maximize cellular spread spectrum capacity," IEEE J. Select. Areas Commun., vol. 13, pp. 1332-1340, Sept. 1995.

[7] A. J. Goldsmith and P. P. Varaiya, "Capacity of fading channels with channel side information," IEEE Trans. Inform. Theory, vol. 43, pp. 1986-1992, Nov. 1997.

[8] T. M. Cover and J. A. Thomas, Elements of Information Theory. New York: Wiley, 1991.

[9] S. Verdú, "Capacity region of Gaussian CDMA channels: The symbolsynchronous case," in Proc. 24th Annu. Allerton Conf. Communications, Control and Computing, Monticello, IL, Oct. 1986, pp. 1025-1034.

[10] R. Knopp and P. A. Humblet, "Information capacity and power control in single-cell multiuser communications," in Proc. IEEE Int. Conf. Communications, Seattle, WA, June 1995, pp. 331-335.

[11] S. Hanly and D. N. C. Tse, "Multiaccess fading channels-Part I: Polymatroid structure, optimal resource allocation, and throughput capacities," IEEE Trans. Inform. Theory, vol. 44, pp. 2796-2815, Nov. 1998.

[12] W. Yu, W. Rhee, and J. M. Cioffi, "Optimal power control in multiple access fading channels with multiple antennas," in Proc. IEEE Int. Conf. Communications, Helsinki, Finland, June 2001, pp. 575-579.

[13] P. Viswanath, D. N. C. Tse, and V. Anantharam, "Asymptotically optimal water-filling in vector multiple-access channels," IEEE Trans. Inform. Theory, vol. 47, pp. 241-267, Jan. 2001.

[14] W. Yu, W. Rhee, S. Boyd, and J. M. Cioffi, "Iterative water-filling for Gaussian vector multiple access channels," in Proc. IEEE Int. Symp. Information Theory, Washington, DC, June 2001, p. 322.

[15] S. Verdú, Multiuser Detection. Cambridge, U.K.: Cambridge Univ. Press, 1998.

[16] E. Biglieri, J. Proakis, and S. Shamai (Shitz), "Fading channels: Information-theoretic and communications aspects," IEEE Trans. Inform. Theory, vol. 44, pp. 2619-2692, Oct. 1998.

[17] S. Shamai (Shitz) and A. D. Wyner, "Information theoretic considerations for symmetric, cellular, multiple-access fading channels-Part I," IEEE Trans. Inform. Theory, vol. 43, pp. 1877-1894, Nov. 1997.

[18] B. Rimoldi and R. Urbanke, "On the structure of the dominant face of multiple-access channels," in Proc. IEEE Information Theory and Communications Workshop, Kruger National Park, South Africa, June 1999, pp. 12-14.

[19] M. J. Panik, Classical Optimization: Foundations and Extensions. Amsterdam, the Netherlands: North-Holland, 1976.

[20] D. P. Bertsekas and J. N. Tsitsiklis, Parallel and Distributed Computation: Numerical Methods. Belmont, MA: Athena Scientific, 1997.

[21] S. Ulukus and R. D. Yates, "Adaptive power control and MMSE interference suppression,” ACM J. Wireless Networks, vol. 4, no. 6, pp. 489-496, Nov. 1998.

[22] S. V. Hanly and D. N. C. Tse, "Resource pooling and effective bandwidths in CDMA networks with multiuser receivers and spatial diversity," IEEE Trans. Inform. Theory, vol. 47, pp. 1328-1351, May 2001.

\section{Optimality and Suboptimality of Multiple-Description Vector Quantization With a Lattice Codebook}

\author{
Chao Tian, Student Member, IEEE, and \\ Sheila S. Hemami, Senior Member, IEEE
}

\begin{abstract}
The asymptotic analysis of multiple-description vector quantization (MDVQ) with a lattice codebook for sources with smooth probability density functions (pdfs) is considered in this correspondence. Goyal et al. observed that as the side distortion decreases and the central distortion correspondingly increases, the quantizer cells farther away from the coarse lattice points shrink in a spatially periodic pattern. In this correspondence, two special classes of index assignments are used along strategic groupings of central quantizer cells to derive a straightforward asymptotic analysis, which provides an analytical explanation for the aforementioned observation. MDVQ with a lattice codebook was shown earlier to be asymptotically optimal in high dimensions, with a curious converging property, that the side quantizers achieve the space filling advantage of an $\boldsymbol{n}$-dimensional sphere instead of an $\boldsymbol{n}$-dimensional optimal polytope. The analysis presented here explains this behavior readily. While central quantizer cells on a uniform lattice are asymptotically optimal in high dimensions, the present authors have shown that by using nonuniform rather than uniform central quantizer cells, the central-side distortion product in an MDSQ can be reduced by $0.4 \mathrm{~dB}$ at asymptotically high rate. The asymptotic analysis derived here partially unifies these previous results in the same framework, though a complete characterization is still beyond reach.
\end{abstract}

Index Terms-Asymptotic analysis, lattice quantization, multiple description, vector quantization.

\section{INTRODUCTION}

In a multiple description (MD) coding scenario, information on the source samples are sent on two channels, each of which may fail. The source coding should provide acceptable reconstruction when only one channel works, and higher quality reconstruction when both channels work. Achieving such multiple descriptions through quantization [2], [4]-[6] can be understood as using two coarse quantizers (the side quantizers) to provide the two descriptions, which can be combined to form a finer quantizer (the central quantizer). Two steps are required: in the first step, the central quantizer functions as a classical quantizer, giving an index $l$. In the second step, the index assignment provides a map from the single index $l$ to an index pair $\alpha(l)=(p, q)$. The indices $p$ and $q$ serve as the two side quantizers' indices, which are transmitted over the two channels.

The MD problem is most often considered in the balanced case, in which the two descriptions are at the same rate and generate the same distortions, and this case is assumed in this correspondence. The distortion generated by each of the two side quantizers is called the side distortion and the distortion generated by the central quantizer is called the central distortion. In the balanced case, rate distortion theory reveals that there is a tradeoff between the side and central distortions [3], [7]. Let mean-squared error be the distortion measure (and it will be the distortion measure adopted in this correspondence). If the side distortion is of the form

$$
D_{1}=b 2^{-2(1-\eta) R}
$$

Manuscript received August 13, 2003; revised April 6, 2004. This work was supported in part by the Multidisciplinary University Research Initiative (MURI) under the Office of Naval Research Contract N00014-00-1-0564.

The authors are with the School of Electrical and Computer Engineering, Cornell University, Ithaca, NY 14853 USA (e-mail: ctian@ece.cornell.edu; hemami@ece.cornell.edu).

Communicated by T. Lindar, Associate Editor for Source Coding.

Digital Object Identifier 10.1109/TIT.2004.834748 